\title{
ORCA (Antarctic Cosmic Ray Observatory): 2018 latitudinal survey
}

\section{J. J. Blanco on behalf of the ORCA team ${ }^{* 1 \dagger}$}

J.J. Blanco ${ }^{1}$, O. García Población ${ }^{1}$, J. I. García Tejedor ${ }^{1}$, C. T. Steigies ${ }^{3}$, J. Medina ${ }^{1}$, M. Prieto $^{1}$, A. López-Comazzi ${ }^{1}$, S. Ayuso ${ }^{1}$, R. Gómez-Herrero ${ }^{1}$, J. A. Garzón ${ }^{2}$, D. García-Castro ${ }^{2}$, P. Cabanelas ${ }^{2}$, A. Gomis-Moreno ${ }^{5}$, V. Villasante-Marcos ${ }^{5}$, B. Heber ${ }^{3}$, Anna Morozova ${ }^{4}$, G.

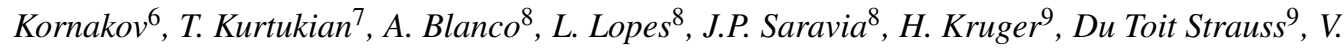
G. Yanke ${ }^{10}$.

A set of detectors devoted to investigate secondary cosmic rays has performed a latitudinal observation from Vigo (Spain) to Juan Carlos I Spanish Antarctic Station (Livingston Island, Antarctic Peninsula) aboard the Sarmiento de Gamboa oceanographic vessel from November $14^{\text {th }}$ to January $2^{\text {nd }}$. The experiment is split into two modules, one composed by a stack of 3NM64, three BF3 bare counters (NEMO) and a muon telescope (MITO) with a mini neutron monitor in a $20^{\prime}$ maritime container on the Sarmiento de Gamboa's deck and a second module (TRISTAN) consisting of a set of 3 RPC planes with a lead layer in between the second and the third plane placed in a separate temperature controlled room below the ship's deck. The complete set of instruments is the Antarctic Cosmic Ray Observatory (ORCA) that has been be installed in the Juan Carlos I Spanish Antarctic Base in Livingston Island (Antarctica). The latitudinal survey took ORCA throughout the South Atlantic magnetic anomaly along the Brazilian coast. ORCA is able to measure fluxes of neutrons of different energies, charged particles (mostly muons) and muon incident directions on the detector surface. In this work, we present the preliminary results of the latitudinal survey.

E-mail: juanjo.blanco@uah.es

${ }^{1}$ U. de Alcalá, Spain. ${ }^{2}$ U. Santiago de Compostela, Spain. ${ }^{3}$ IEAP, Christian Albrechts Universität zu Kiel, Germany. ${ }^{4}$ CITEUC, Coimbra, Portugal. ${ }^{5}$ Instituto GeogrÃąfico Nacional, Madrid, Spain. ${ }^{6}$ TU-Darmstadt, Germany. ${ }^{7}$ CEN-Bordeaux, France. ${ }^{8}$ LIP-Coimbra, Portugal. ${ }^{9}$ Center for Space Research, North-West University, South Africa. ${ }^{10}$ Institute of Terrestrial Magnetism, Ionosphere and Radio Wave Propagation RAS (IZMIRAN), Moscow, Russia

36th International Cosmic Ray Conference -ICRC2019-

July 24th - August 1st, 2019

Madison, WI, U.S.A.

\footnotetext{
* Speaker.

${ }^{\dagger}$ Thanks to project CTM2016-77325-C2-1-P and CTM2016-77325-C2-2-P funded by Ministerio de Economía y Competitividad and by the European Regional Development Fund, FEDER.
} 


\section{Introduction}

Galactic cosmic rays arrive at the Earth as an isotropic flux at the border between the magnetosphere and the solar wind. From this border to ground level, the magnetosphere sets different rigidity thresholds depending on the location of the stations devoted to the cosmic ray observation. This threshold is lower for stations close to the magnetic poles and higher near the magnetic equator. This geographic response allows one to build a global detector compose of similar instruments distributed around the world. Maybe, the most frequently used instrument for this kind of measurements is the neutron monitor.

A neutron monitor is a ground-based instrument designed to measure secondary neutrons produced by the interaction of cosmic rays and solar energetic particles with the atmospheric molecules [1]. Many of these neutron monitors upload their measurements to the Neutron Monitor Data Base (NMDB) and data is accessible through the web page http://www. nmdb.eu/nest/. Because of its high stability and long term measurements with little maintenance work, a neutron monitor is one of the most trustworthy detector for monitoring cosmic ray variations.

Fast latitudinal surveys are a direct approach to the observation of the rigidity dependence of cosmic rays observed at ground level. Fast means a complete survey in a few weeks to reduce the variation of cosmic ray flux because of the solar cycle. This survey is even more effective if neither Fobush decreases nor ground level enhancements happen during the survey. There are many examples of latitudinal cosmic ray surveys, for instance a historical review is done by [2] or a study of the cosmic ray modulation along latitude surveys at different solar cycle epochs is shown in [3]. The usual instrument used for performing latitude surveys is a neutron monitor and in some occasions is complemented with a mini neutron monitor [4].

As far as we know, this is the first time that a latitudinal survey is performed by using an instrument composed of a neutron monitor (NEMO), a muon telescope (MITO), a mini neutron monitor and Resistive Plate Chamber (RPC) based detector; all of them integrated into the Antarctic Cosmic Ray Observatory (ORCA). In this work, we describe the detector ORCA and the survey conditions with the preliminary observations obtained during the survey aboard the Spanish research vessel Sarmiento de Gamboa.

\section{Latitudinal survey}

The Spanish research vessel Sarmiento de Gamboa is one of the two research vessels that can participate in the Spanish Antarctic campaign. Recently, Sarmiento de Gamboa participated in the latest campaign from November 2018 to April 2019. This was the first time that a cosmic ray detector was operational aboard performing a latitudinal survey from $\mathrm{N} 42^{\circ}$ to $\mathrm{S} 62^{\circ}$ during a Spanish campaign. The Antarctic Cosmic Ray Observatory (ORCA) was split in two sets, one into a transport container on the stern deck and the other in a thermally controlled room below the deck. The Spanish research vessel Sarmiento de Gamboa traveled from Vigo (Spain) to Juan Carlos I Antarctic Base between $16^{\text {th }}$ November 2018 to $2^{\text {nd }}$ January 2019. Its route took it from Vigo to Punta Arenas (Chile, $12^{\text {th }}$ December) through the Canary Islands, the Brazilian coast and so under the South Atlantic Anomaly (SSA), the Argentinian coast and the Strait of Magellan. Sarmiento de Gamboa stopped for four days and followed its course through Ushuaia (Argentina) to Juan 
Carlos I Spanish Antarctic Base arriving at the base on $27^{\text {th }}$ December. The vessel comes back to Ushuaia and returns to Juan Carlos I finishing the observation period on $2^{\text {nd }}$ January 2019 when the cosmic ray detector was switched off to be installed at the Juan Carlos I station. A map with the Sarmiento de Gamboa's course (white line) can be seen in figure 1. A picture of the container (the black container below the blue one) on the stern deck is shown in figure 2. Most of ORCA's sensors were continuously measuring in a latitudinal survey as wide as $104^{\circ}$ in one way scan and TRISTAN worked along a two way scan of $96^{\circ}$ (Table 1). The scans of the two way scan separated by two months.

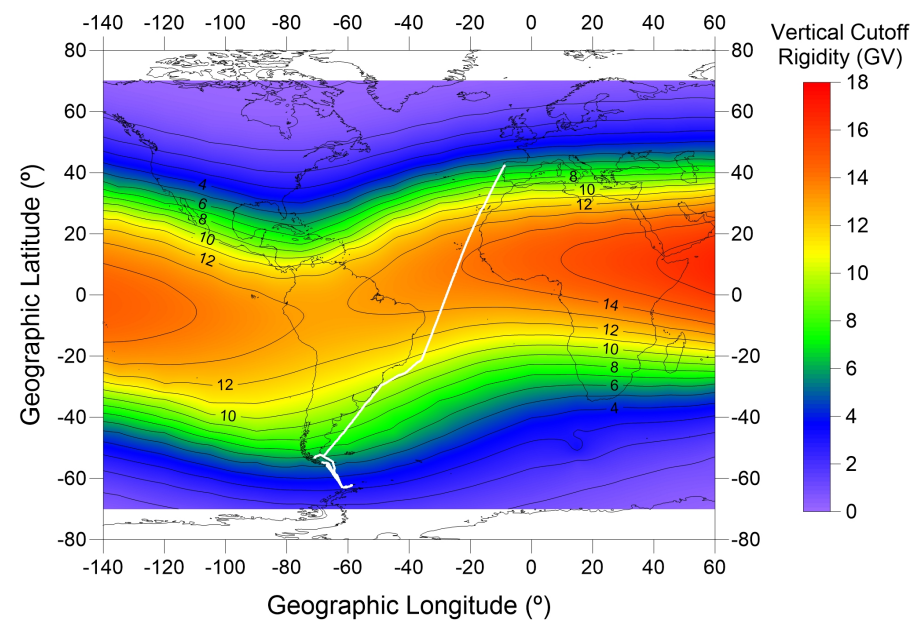

Figure 1: Sarmiento de Gamboa's course from $16^{\text {th }}$ November 2018 to $2^{\text {nd }}$ January 2019. Vertical cutoff rigidities for 1980 [5].

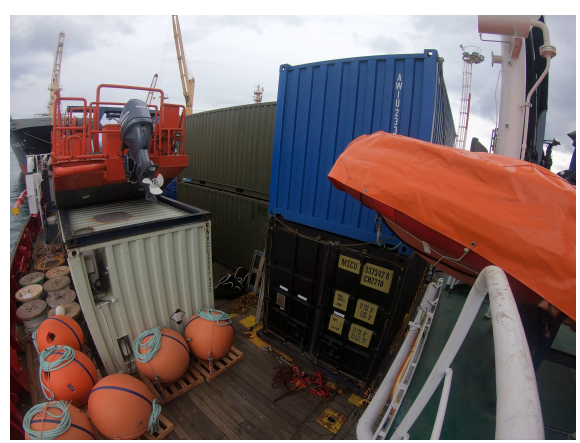

Figure 2: $\mathrm{ORCA}^{\prime} \mathrm{s}$ container (the black one below the blue one) aboard Sarmiento de Gamboa

\section{Antarctic Cosmic Ray Observatory (ORCA)}

ORCA is an instrument made by a set of detectors to measure different populations of secondary cosmic rays at ground level. It is divided into two main blocks, one of them is composed of a neutron detector (NEMO) a muon telescope (MITO) and a mini neutron monitor installed in a 20 feet container which is thermally isolated and environmentally controlled (see figure 3 ). The pressure, temperature and humidity is recorded by a Vaisala PTU300 Combined Pressure, Humidity and Temperature Transmitter inside the container. The Vaisala sensor is at the bottom of the electronics rack (figure 3). This block is already operative in Juan Carlos I Spanish Antarctic Base [6]. The second block is composed of a single detector based on RPCs named TRISTAN, to measure charge particles and photons. TRISTAN was operated in a temperature controlled room under the Sarmiento deck (figure 4). Outdoor temperature, pressure, ship position and more variables about the ship's status are provided by Sarmiento de Gamboa instruments. The ORCA instruments measured fluxes of secondary cosmic rays for 48 days along a latitudinal survey covering more that 100 degrees between Vigo (Spain) and Livingston Island (Antarctic peninsula).

\subsection{NEMO}

The Neutron Monitor (NEMO) is a neutron monitor with two groups of proportional detectors placed on top of each other. The lower group is formed by three BP-28 detectors in the typical con- 


\begin{tabular}{|c|c|c|c|c|c|c|}
\hline Sensor & $\begin{array}{l}\text { Real } \\
\text { Time }\end{array}$ & $\begin{array}{l}\text { Directional } \\
\text { information }\end{array}$ & $\begin{array}{l}\text { Particle } \\
\text { detection }\end{array}$ & $\begin{array}{c}\text { Environmental } \\
\text { corrections }\end{array}$ & $\begin{array}{l}\text { Data } \\
\text { survey }\end{array}$ & Trip \\
\hline NEMO & No & No & $\mathrm{n}$ & Yes: $\mathrm{P}$ & $\mathrm{N} 42^{\circ}$ to $\mathrm{S} 62^{\circ}$ & One way \\
\hline MITO & No & Yes & $\mu$ & Yes: P/T/T ${ }_{l a b}$ & $\mathrm{~N} 42^{\circ}$ to $\mathrm{S} 62^{\circ}$ & One way \\
\hline TRISTAN & No & Yes & $\mu, \mathrm{e}-, \gamma$ & Yes: P/T/T ${ }_{l a b}$ & $\mathrm{~N} 42^{\circ}$ to $\mathrm{S} 54^{\circ}$ & Two ways \\
\hline Mini-NM & No & No & $\mathrm{n}$ & Yes: $\mathrm{P} / \mathrm{T} / \mathrm{T}_{l a b}$ & $\mathrm{~N} 42^{\circ}$ to $\mathrm{S} 62^{\circ}$ & One way \\
\hline Vaisala & No & No & $\mathrm{P}, \mathrm{T}, \mathrm{RH}$ & No & $\mathrm{N} 42^{\circ}$ to $\mathrm{S} 62^{\circ}$ & One way \\
\hline
\end{tabular}

Table 1: ORCA sensors

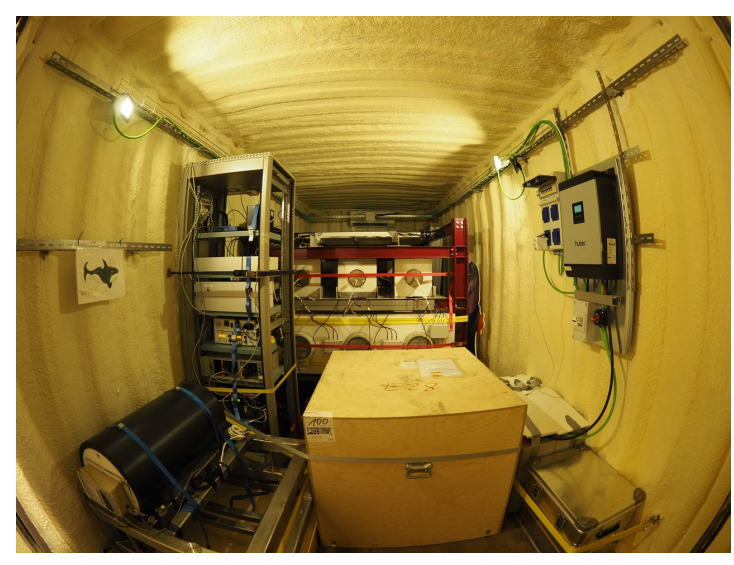

Figure 3: NEMO, MITO and mini neutron monitor in the container on Sarmiento de Gamboa's deck.

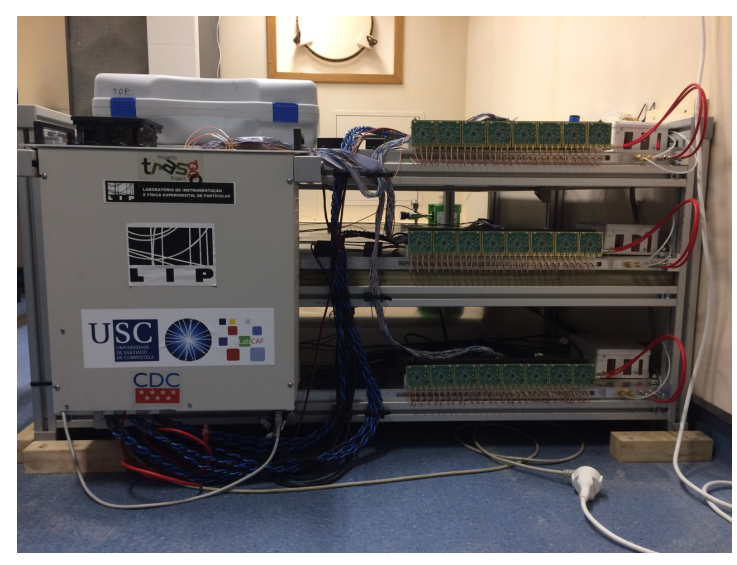

Figure 4: TRISTAN in the thermally controlled room under Sarmiento de Gamboa's deck.

figuration of a 3NM64 type neutron monitor, that is, with an outer reflector made of polyethylene, with lead producers in the form of lead rings around the moderator, a moderator also of polyethylene and finally a BP28 type detector tube. On the other hand, the upper group consists of three bare detector tubes (3BNM) of the type LND2061. The signals from the detectors are preprocessed in a signal conditioning system before reaching the data acquisition system. This system consists of an FPGA with an IP core specifically designed for this application, and an embedded Linux Beaglebone Black system in which the capture code is executed, as well as the necessary corrections, the editing of the data and its subsequent publication in a database. This system keeps its local clock synchronized using an NTP server equipped with a GPS receiver. NEMO measures neutrons at two different energy thresholds thanks to two sets of three $\mathrm{BF}_{3}$ gas filled tubes, one set surrounded by a $5 \mathrm{~cm}$ thick lead ring and the other bare tubes. One minute neutron count rates are measured continuously. Both the 3NM64 and 3BNM detectors produce one minute data including those from the meteorologic station, i.e. pressure, room temperature and relative humidity.

\subsection{MITO}

The Muon Impact-Tracer Observer (MITO) [7] is a telescope made by a stack of two (MITOtop and MITO-bottom) BC-400 organic scintillators $(100 \mathrm{~cm}$ x $100 \mathrm{~cm}$ x $5 \mathrm{~cm}$, poly-vinyl-toluene with $65 \%$ anthracene). Four photomultipliers (PMTs) are coupled to each scintillator by means of a pyramidal light guide. Each PMT collects the light reaching the lateral surface and generates a 
pulse which carries information about the distance between the particle track and the corresponding lateral surface of the BC-400 scintillator. Track impact is calculated by comparison of the pulse height detected in the PMTs when they are working in coincidence (see figure 5 and figure 6). The two scintillators impact points give us information about the muon incident direction. Both scintillators are placed at the top and bottom of a metallic structure $136,5 \mathrm{~cm}$ apart of each other with a 10 $\mathrm{cm}$ lead layer in between (this is the lead in the 3NM64 module). MITO has two data acquisition

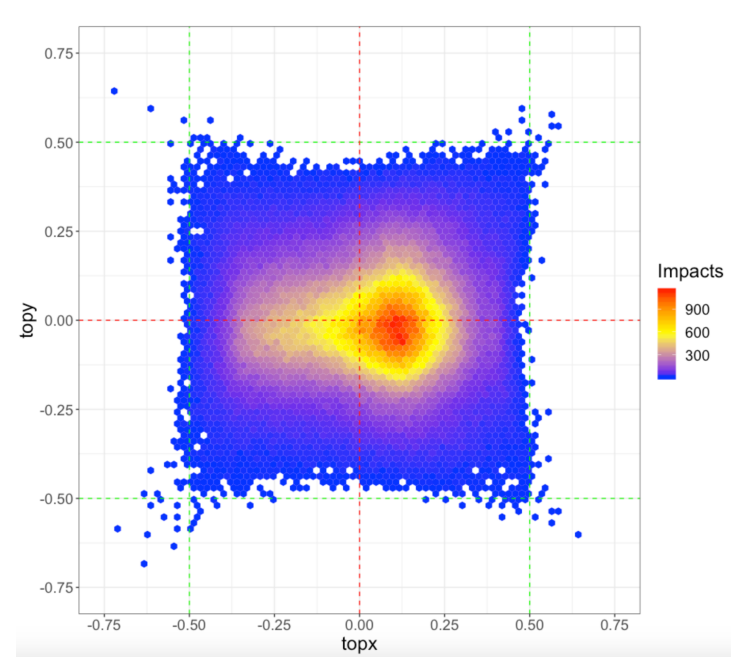

Figure 5: MITO-Top. Histogram of impact points for December $6^{\text {th }}$. X and $\mathrm{Y}$ axis are in meters.

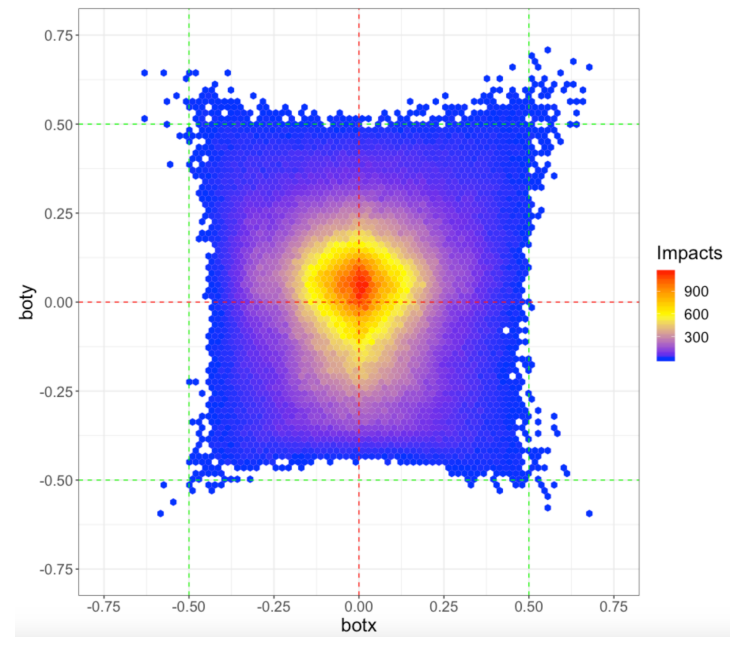

Figure 6: MITO-Bottom. Histogram of impact points for December $6^{\text {th }}$. $\mathrm{X}$ and $\mathrm{Y}$ axis are in meters.

systems operating in parallel, SAS and Aracne. SAS counts the impacts on the scintillator keeping those that follow four different coincidence channels, Top: the four PMTs in the upper scintillator, Bottom: the four PMTs in the bottom scintillator, coin8: the eight PMTs, i.e. particles that cross both scintillators, and Lateral: a combination of two PMTs in the upper scintillator located at a common lateral side and two PMTs in the bottom scintillator but at the opposite lateral side of the previous ones. Aracne records the highest pulse of all the PMTs gathered in a particle detection. From them, it can select the events under the same coincidence channels as SAS. The high pulse analysis allows one to determine the impact point on the scintillators and with the two impact points the particle trajectory throughout MITO can be reconstructed. An example of a histogram showing the impact point on one of the MITO scintillators can be seen in figure 6 and figure 5. Additionally, Aracne has an operational mode that allows to record the complete pulse shape from all the PMTs. MITO is operating in a one minute counting mode with four coincidence channels and an event to event storage of the pulse height recorded in every PMT simultaneously with a time resolution of $25 \mathrm{~ns}$.

\subsection{TRISTAN}

The TRISTAN detector is a detector of the TRAGALDABAS family [8]. It has been designed to be part of ORCA. It is composed by three planes with a mosaic of RPCs quite similar to those of the TRAGALDABAS detector. In TRISTAN each plane is read out by 30 rectangular pads and behind the second layer there is a $1 \mathrm{~cm}$-thick lead plate covering around a $60 \%$ of the surface of 
the plane. The aim of this layer is to induce the growth of the electromagnetic showers associated either to electrons or gammas. TRISTAN also works in an event to event approach with a time resolution of $280 \mathrm{ps}$. It can trace the particle path by combining the pad signals which give a coincidence measurement in the different TRISTAN planes. With a combination of coincidence detection and multiplicity measurements can identify muon, electron and gamma rays giving an angular resolution for incident particles of $\sim 10^{\circ}$.

\subsection{Mini neutron monitor}

These kind of detectors are called calibration neutron monitors [9], they have a total length of $753 \mathrm{~mm}$ and a mass of $223 \mathrm{~kg}$; see left front corner in figure 3. They are therefore mobile, and are readily transported to almost any location around the world as a unit, so that it is not necessary to dismantle them, which usually causes changes in detection efficiency. The mini neutron monitor has a pressure sensor and GPS integrated. The one which travels with ORCA is filled with 2044 $\mathrm{mmHg}{ }^{3} \mathrm{He}$ gas. The mini neutron monitor as well as MITO and TRISTAN records event to event giving information about counting rate and multiplicity in 1-min averages. Its expected counting rate is about 84 counts/s at sea level and cutoff rigidity of $1 \mathrm{GV}$. Nevertheless, an abnormally low counting rate with jumps of the baseline of the data leads us to believe that the measurements of the mini neutron monitor are not valid. This is most likely due to damage of the high voltage supply during shipping, although environmental effect cannot be ruled out.

\section{Observations and preliminary results}

ORCA has performed a latitudinal scan from $16^{\text {th }}$ November 2018 to $2^{\text {nd }}$ January 2019. Along this period, no major solar events were observed and as such a very low effect on cosmic ray modulation due to short term solar activity is expected. The observation shown in this work are non-corrected measurements. This means that atmospheric (pressure, temperature) and rigidity corrections have not been included. These kinds of corrections have to be taken into account [10]. Nevertheless, the aim here is to show the major effects of the geomagnetic field on particle intensities during the survey and uncorrected measurements are good enough for this purpose.

Figure 7 shows the variation of the observed counting rates along the survey. Only NEMO and MITO were fully operative along the trip. We must say that Aracne, one of the acquisition systems of MITO, started its operation on November $26^{\text {th }}$. TRISTAN was working from $16^{\text {th }}$ November 2018 to $11^{\text {nd }}$ December 2018. The effect of the magnetosphere is clearly visible in all the sensors integrated in ORCA. The clearest effect is on neutrons as can be seen in NEMO data, TRISTAN and MITO-Top show also a clear decrease in coincidence with the maximum in rigidity. We have to point out that while the minimum in neutrons is very close to the rigidity maximum, TRISTAN and MITO-Top have their minimum shifted to south with respect rigidity. MITO-Bottom, still shows a rigidity effect but weaker than the observed by MITO-Top. We should remember that between Top and Bottom there is a $10 \mathrm{~cm}$ lead layer that prevents the entrance of less energetic particles like electrons or gammas. The response to the rigidity is shown in figure 8. Although no atmospheric corrections has been done, the dependence of counting rates with respect to rigidity emerges clearly for all the ORCA's instruments. A very rude computation of the variation rate of countrate over rigidity gives the following rates in (counts $\left.\cdot \mathrm{min}^{-1} \cdot G V^{-1}\right)$ : -0.083 (3NM64), 


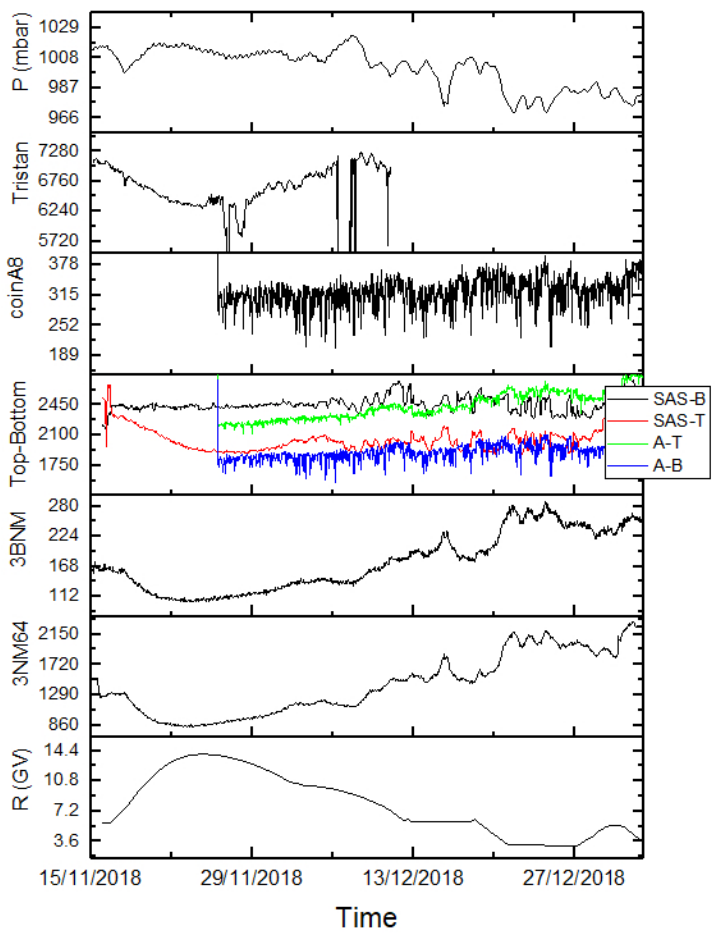

Figure 7: Hourly averaged data along the survey. From top to bottom: pressure in mbar, TRISTAN, MITO-coin8, MITO-Top and MITO-Bottom, NEMO-3BNM, NEMO-3NM64 counting rates in counts per minute and cutoff rigidity $(\mathrm{GV})$.

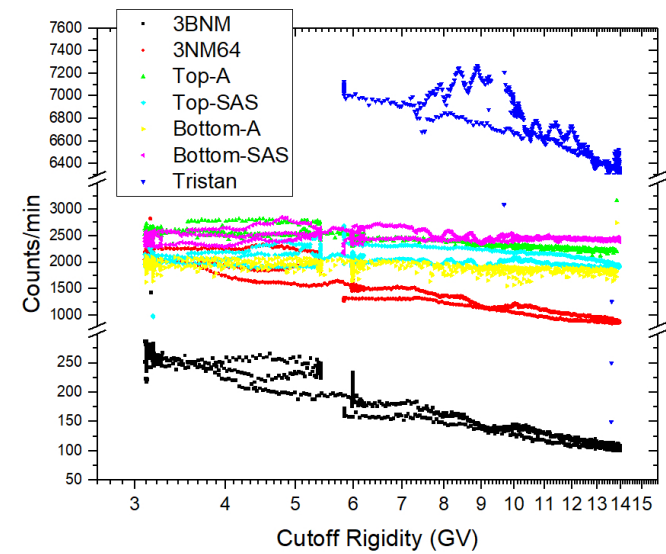

Figure 8: Hourly averaged counting rates vs rigidity. NEMO 3NM64 and 3BNM, MITO top and bottom and TRISTAN.

-0.079 (3BNM), -0.020 (MITO-Top (SAS)),-0.017 (MITO-Top (Aracne)), -0.014 (TRISTAN), 0.011 (MITO-Bottom(Aracne)) and -0.002 (MITO-Bottom(SAS)). The response with rigidity is different for NEMO and MITO-TRISTAN. Assuming that less energetic primary cosmic rays on the top of the magnetosphere are more affected by magnetic field than more energetic cosmic rays, we can say that neutrons measured by NEMO are produced by primary cosmic rays with less energy, on average, than those that produce the particles, mostly muons but also electrons, protons and gammas, detected by MITO and TRISTAN.

\section{5. conclusions}

This work presents the preliminary results for the latitudinal scan performed by the Antarctic Cosmic Ray Observatory (ORCA). ORCA's instruments measured counting rates of neutrons and muons mainly along a latitudinal survey from $\mathrm{N} 42^{\circ}$ to $\mathrm{S} 62^{\circ}$. Although no atmospheric and rigidity corrections have been performed yet, some preliminary conclusions can be reported:

- Neutrons are produced by less energetic primary cosmic rays than muons.

- Nevertheless, both neutrons and muons show a rigidity dependence. 
- The last result could confirm that the flux of primary cosmic rays with rigidities around 14 $\mathrm{GV}$ can contribute to the muon flux observed at sea level.

Some outstanding issues have to be addressed in future work, including the correction of the data, a comparison with models and a more careful inspection of the effect of the South Atlantic Anomaly on secondary cosmic rays at sea level.

\section{References}

[1] John A. Simpson. The cosmic ray nucleonic component: The invention and scientific uses of the neutron monitor - (keynote lecture). Space Science Reviews, 93(1):11-32, Jul 2000.

[2] L.I. Dorman. Cosmic Ray Planetary Surveys on Ships, Trains, Tracks, Planes, Balloons, and Satellites, pages 191-340. Springer Netherlands, Dordrecht, 2009.

[3] W. Nuntiyakul, P. Evenson, D. Ruffolo, A. Sáiz, J. W. Bieber, J. Clem, R. Pyle, M. L. Duldig, and J. E. Humble. Latitude survey investigation of galactic cosmic ray solar modulation during 1994-2007. The Astrophysical Journal, 795(1):11, oct 2014.

[4] H. Krüger, H. Moraal, J. W. Bieber, J. M. Clem, P. A. Evenson, K. R. Pyle, M. Duldig, and J. E. Humble. Latitude surveys with a calibration neutron monitor. International Cosmic Ray Conference, 2:473, Jan 2005.

[5] D. F. Smart and M. A. Shea. Galactic Cosmic Radiation and Solar Energetic Particles, volume 27, chapter 6, pages 6.1-6.29. Adolph S. Jursa, AIR FORCE GEOPHYSICS LABORATORY, Apr 1988.

[6] J.J. Blanco, O. García Población, J.I. García Tejedor, J. Medina, M. Prieto, A. López-Comazzi, S. Ayuso, and R. Gómez-Herrero. A new neutron monitor at the Juan Carlos I Spanish Antarctic Station. POS ( ICRC2019) 1060. In P. Desiati, T. Gaisser, and A Karle, editors, 36th International Cosmic Ray Conference (ICRC2019), volume 1 of Proceedings of Science, July 2019.

[7] S. Ayuso, J.I. García Tejedor, J.J. Blanco, R. Gómez-Herrero, J. Medina, and O. García Población. MITO, a new directional muon telescope design. First observations. PoS ( ICRC2019) 180. In P. Desiati, T. Gaisser, and A Karle, editors, 36th International Cosmic Ray Conference (ICRC2019), volume 1 of Proceedings of Science, July 2019.

[8] H Alvarez-Pol, A Blanco, J J Blanco, J Collazo, P Fonte, J A Garzón, A Gómez, G Kornakov, T Kurtukian, L Lopes, M Morales, A Morozova, M A Pais, M Palka, V Pérez Muñuzuri, P Rey, P Ribeiro, M Seco, and J Taboada. TRAGALDABAS: A new high resolution detector for the regular study of cosmic rays. Journal of Physics: Conference Series, 632:012010, aug 2015.

[9] H. Krüger, H. Moraal, R. Nel, H. G. Krüger, and M. O’Kennedy. The mini neutron monitor programme. In A. S. Borisov, V. G. Denisova, Z. M. Guseva, E. A. Kanevskaya, M. G. Kogan, A. E. Morozov, V. S. Puchkov, S. E. Pyatovsky, G. P. Shoziyoev, M. D. Smirnova, A. V. Vargasov, V. I. Galkin, S. I. Nazarov, and R. A. Mukhamedshin, editors, 34th International Cosmic Ray Conference (ICRC2015), volume 34 of International Cosmic Ray Conference, page 223, July 2015.

[10] J. M. Clem, J. W. Bieber, P. Evenson, D. Hall, J. E. Humble, and M. Duldig. Contribution of obliquely incident particles to neutron monitor counting rate. Journal of Geophysical Research: Space Physics, 102(A12):26919-26926, 1997. 